\title{
Repenser la neutralité axiologique
}

Objectivité, autonomie et délibération publique

Rethinking the axiological neutrality: objectivity, autonomy and public

deliberation

\section{Marc-Kevin Daoust}

\section{OpenEdition \\ Journals}

\section{Édition électronique}

URL : http://journals.openedition.org/ress/3000

DOl : $10.4000 /$ ress. 3000

ISSN : $1663-4446$

\section{Éditeur}

Librairie Droz

\section{Édition imprimée}

Date de publication : 15 mai 2015

Pagination : 199-225

ISSN : 0048-8046

\section{Référence électronique}

Marc-Kevin Daoust, « Repenser la neutralité axiologique », Revue européenne des sciences sociales [En ligne], 53-1 | 2015, mis en ligne le 01 janvier 2019, consulté le 02 mai 2019. URL : http:// journals.openedition.org/ress/3000; DOI : 10.4000/ress.3000 


\title{
REPENSER LA NEUTRALITÉ AXIOLOGIQUE OBJECTIVITÉ, AUTONOMIE ET DÉLIBÉRATION PUBLIQUE
}

\author{
MARC-KEVIN DAOUST \\ Université de Montréal \\ marc-kevin.daoust@umontreal.ca
}

\begin{abstract}
Résumé. L'objectif de cet article est double. D'une part, il vise à identifier une interprétation éthique de la neutralité axiologique, et non de réduire ce critère à des considérations épistémologiques comme la distinction entre faits et valeurs. On peut, en effet, interpréter le critère de neutralité axiologique comme un mécanisme visant à défendre l'autonomie des différents membres de la communauté universitaire. D'autre part, cet article entend utiliser cette interprétation éthique pour répondre aux critiques contemporaines de la neutralité axiologique. Amartya Sen et Hilary Putnam ont proposé des critiques pertinentes de la neutralité axiologique, en réfléchissant sur les conditions d'objectivité du discours éthique et politique. Au terme de cette analyse, nous proposerons une conception publique de la science, qui répond à l'impératif éthique de neutralité en sciences sociales, ainsi qu'au programme de recherche amorcé par Sen et Putnam. L'examen critique et public de la science est ce qui permet de respecter tant l'exigence de neutralité chez Weber que la demande d'une science renouvelée chez Sen et Putnam.
\end{abstract}

Mots-clés: autonomie, concepts éthiques denses, objectivité, neutralité axiologique.

\begin{abstract}
This article has two goals. My first objective is to understand axiological neutrality, not as an epistemological requirement, but rather as an ethical requirement. Weber claims that it would be contrary to the principle of autonomy to allow the professor to influence and convince students, in a biased manner, as well as to adhere to certain normative themes. Based on this interpretation, my second objective is to answer contemporary criticism of axiological neutrality. Amartya Sen and Hilary Putnam reject the so-called subjectivity of values and, by doing this, defend an enriched view of science where ethical values are present. To answer Sen and Putnam's arguments, I defend a public conception of science. From this point of view, I respect both the requirement of a value-free science and the new model of science presented by Sen and Putnam. A public conception of science makes sure that all conditions are present so that a normative discussion truly results in objectivity.
\end{abstract}

Keywords: autonomy, axiological neutrality, objectivity, thick ethical concepts. 
Dans quelles limites un scientifique est-il justifié d'user de son autorité, surtout quant aux questions éthiques et politiques? Peut-on imaginer des conditions dans lesquelles il serait préférable que le ou la scientifique limite l'usage de son autorité, qu'il s'en tienne à une position «neutre»? Comme le souligne Nathalie Heinich à juste titre, «la règle wébérienne de "neutralité axiologique”, prônant l'abstention de tout jugement de valeurs par l'enseignant ou le chercheur dans le cadre de ses fonctions, est loin de faire aujourd'hui, concrètement, l'unanimité » (2006, p. 290). Le but de cette analyse est double. D’une part, l'objectif poursuivi est d'analyser les limites de l'autorité scientifique, en particulier lorsqu'il s'agit d'élucider des questions d'éthique et de philosophie politique. Il s'agit de déterminer, au regard de l'œuvre de Max Weber, s'il existe des raisons suffisantes appuyant le critère de neutralité axiologique. L'analyse en cours vise à formuler une thèse d'inspiration wébérienne, plutôt qu'une interprétation exacte et décisive du critère de neutralité axiologique.

Max Weber est un auteur incontournable pour analyser les limites de l'autorité du scientifique, notamment parce qu'il analyse les conditions de possibilité du discours normatif. Notre hypothèse de départ, développée à partir de l'œuvre de Weber, est qu'on ne peut réfléchir sur l'éthique et la philosophie politique sans d'abord considérer les conditions dans lesquelles le discours éthique est possible. Un amphithéâtre universitaire où le professeur exerce une forte autorité - par exemple, en exigeant la présence, le silence et l'écoute des étudiants - n'est pas un lieu propice à une discussion normative rationnelle. En effet, dans ces conditions, aucun examen rationnel et critique des thèses présentées n'est possible, notamment parce que le professeur utilise ses ressources pour empêcher cet examen. En ce sens, par respect pour l'éthique et la philosophie politique, le professeur doit se conformer à certains paramètres lorsqu'il mène une discussion normative.

Cette interprétation nous permet de comprendre des problèmes particuliers, comme l'enseignement d'un cours magistral. Il n'en demeure pas moins que, comme le propos de Weber est plutôt «concret», il s'applique difficilement à une gamme générale de problématiques. C’est pourquoi nous proposons de systématiser l'approche de Weber. Être professeur sera alors abordé comme 
une fonction, un poste où l'individu agit suivant l'usage privé de la raison. L'usage de la raison est privé, en ce sens qu’un professeur doit obéir aux règles de la communauté morale universitaire, et donc se priver de certaines libertés. En échange de cet usage privé, l'individu se voit conférer des instruments et des ressources pour mener ses différentes tâches de professeur à terme. Grâce à cette approche théorique, nous pourrons déterminer quel est l'usage légitime de la raison dans diverses circonstances générales, notamment dans les séminaires, les publications scientifiques, les colloques et l'embauche.

Cet article serait incomplet sans «confronter» cette interprétation de la neutralité axiologique à des postures critiques contemporaines. Plusieurs auteurs citent l'analyse anti-positiviste d'Hilary Putnam pour rejeter le critère de neutralité éthique. En effet, Putnam montre, dans Fait-valeur: la fin d'un dogme et autres essais (2004 [2002]), que la thèse positiviste selon laquelle les faits sont objectifs et les valeurs subjectives ne repose sur aucun argument cohérent. Comme la science présuppose des valeurs épistémiques, affirmer que les valeurs sont subjectives revient à prétendre que la science est subjective. Putnam vise alors à montrer que les valeurs peuvent tendre à une forme d'objectivité assouplie. Le fait qu'une thèse normative soit comparée, critiquée, amendée et étoffée lui confère une forme d'«objectivité assouplie». Sen reprend l'argument de Putnam et montre que l'étude à la fois descriptive et normative présente un potentiel épistémique manifeste. Les approches «denses » (à la fois descriptives et normatives) auraient, toujours selon Sen, un meilleur potentiel de prédictibilité, de fécondité et de pertinence. Comme la science se développe à partir de ce potentiel, ce serait donc en vertu d'un intérêt proprement scientifique que l'on devrait écarter la neutralité axiologique.

Alors, comment concilier les approches «denses » au respect de la neutralité axiologique? Nous proposerons le développement d’une science publique, à la fois descriptive et normative, qui respecte les conditions d'objectivité assouplie de l'éthique. En revanche, cette science, de par sa nature dialogique, respecte aussi notre interprétation de la neutralité axiologique. Ainsi, tout en suivant le fil logique développé par Putnam, nous défendrons qu'il existe des raisons valables de préserver le critère de neutralité axiologique. 
En définitive, l'intérêt de cet article est de proposer une analyse philosophique du critère de neutralité axiologique. Ce critère est souvent critiqué en fonction d'interprétations étroites. Il existe pourtant des raisons philosophiques fondamentales d'adhérer à un tel critère. Le but de cet article est donc d'en proposer une lecture riche et intéressante et de l'affiner grâce à la critique de Putnam.

\section{DEUX INTERPRÉTATIONS DE LA NEUTRALITÉ AXIOLOGIQUE}

Que signifie le fait d'éviter la formulation de jugements de valeur dans les résultats de recherche? D’abord, il y a un sens faible quant à ce que signifie minimalement la neutralité éthique'. Pour Weber, le chercheur ne peut pas fausser ses données, modifier ses résultats de recherche pour accommoder ses propres idéaux normatifs (Bowen, 1977, p. 20I) : «one does not prejudge the outcome of inquiry before the results are in » (ibid., p. 205). Instrumentaliser les faits n'est pas cohérent avec le principal objectif de la science, soit d'établir une connaissance fiable des phénomènes observables (Dussault, 2008, p. 33 ; Hennis, 1994, p. I22; Weber, 1965 [1917], p. 96). La neutralité éthique de Weber va toutefois beaucoup plus loin: elle interdit l'enseignement, sur la base de travaux de recherche, des jugements de valeur. Pour Weber, le chercheur doit s'en tenir à une description des faits, et éviter les jugements évaluatifs (Weber, 1963 [1919], p. 494-498). L’effet immédiat de la neutralité axiologique est d'exiger du chercheur qu'il agisse comme s'il était sceptique ou apolitique, distant face à toute thèse normative² (Miller, 1979, p. 242).

If we take the sphere of scientific inquiry as our focal point, the first of these asymmetrical demands emphasizes the possibilities of scientific activity, when it is conducted without being disturbed by practical value judgments, while the second asymmetrical demand stresses the fact that science has limits which it cannot, and therefore should not pretend to be able to, overstep (Bruun, 2007, p. 75).

I Dans cette analyse, nous laissons de côté le débat sur l'appellation du critère. «Neutralité axiologique» est l'expression la plus répandue, alors que «neutralité éthique» reflète mieux le sens de ce critère. Dans le cadre de cette analyse, nous utiliserons ces deux expressions comme des synonymes.

2 Au minimum, la classe devrait être en mesure de distinguer le moment où le professeur émet des jugements de fait, et le moment où il émet des jugements de valeur. Au mieux, le professeur devrait s'en tenir aux jugements de fait (Miller, 1979, p. 243). 
La neutralité axiologique ne concerne pas les débats sur la place publique. Le lieu où s'exprime un professeur est donc très important. L'exigence de neutralité de Weber concerne uniquement le domaine académique, comme l'enseignement et la recherche (McCarthy, 200I, p. I48). Dans les lieux politiques où les intervenants sont en situation d'autonomie ${ }^{3}$, les chercheurs peuvent jouer un tout autre rôle social (Weber, 1965 [1917], p. 478). Le professeur doit s'abstenir de toute évaluation normative en classe, mais sur la «place publique», il peut intervenir et alimenter le débat. Catherine Colliot-Thélène résume cette idée dans le passage suivant: «[Weber] souligne tout d'abord [...] que cette interprétation de la «vertu» professorale présuppose un cadre institutionnel bien précis. C'est du cours magistral qu'il est question» (2003, p. 33).

\section{I.I. UN BREF RETOUR SUR L'INTERPRÉTATION STRAUSSIENNE DE LA NEUTRALITÉ AXIOLOGIQUE}

Il existe un courant interprétatif - souvent associé à Leo Strauss - selon lequel Weber défendrait ici une forme de dichotomie entre les faits et les valeurs. Selon cette interprétation, Weber trouverait dans les jugements de fait des énoncés objectifs, sur lesquels peuvent reposer la science et tout discours cherchant à atteindre une certaine forme d'objectivité. D’un autre côté, les jugements de valeur constitueraient des énoncés subjectifs, des énoncés relatifs à chaque agent qui n’auraient, en définitive, aucune condition d'existence objective. Ainsi, Strauss associera Weber au courant nihiliste, voire à une forme de décisionnisme (Behnegar, 1997, p. I00; Strauss, 1999, p. 42).

Sans entrer dans le détail de cette interprétation et de ses qualités ${ }^{4}$, quelques remarques s'imposent. Il est vrai que, pour Weber, les valeurs se forment à l'extérieur des sciences, comprises comme les disciplines empiriques basées sur l'observation. Les valeurs sont importantes dans l'entreprise scientifique, mais

3 Par situation d'autonomie, on fait référence aux lieux où les participants sont libres de participer au débat, d'intervenir, qu'ils ont la possibilité complète d'exprimer sa pensée. II s'agit de lieux où l'influence et le contrôle institutionnel sont limités.

4 Pour approfondir cette interprétation et ses qualités, on peut consulter Gonthier (2006) et Heinich (2006) qui défendent deux interprétations opposées sur la question. 
de manière extrinsèque (Segady, I987, p. 35). Or, cela ne signifie pas, comme le croit Strauss, que Weber adhère au nihilisme des valeurs (Myers, 2005; Portis, 1980, p. 415-416). Weber l'indique clairement dans le passage suivant: «[J]e voudrais m'élever contre la prétention des partisans de la neutralité axiologique qui voient dans le simple fait des variations historiques et singulières [...] une preuve en faveur du caractère inévitablement "subjectif" de la morale » (1965 [1917], p.487). Loin d'associer les jugements factuels à l'objectivité et les jugements évaluatifs à la subjectivité, Weber accorde plutôt une pertinence fondamentale au discours normatif. «Of all the major misunderstandings claim that Weber's of his postulate, one finds at its core the issue of a "methodological" postulate leads to indifference and value nihilism » (Hennis, 1994, p. I13).

Weber reconnaît plutôt qu'il existe plusieurs types de discours rationnels, dont la philosophie éthique et politique fait partie. Comme ces deux discours n'ont pas les mêmes exigences de validité, il est possible de confondre l'éthique avec des résultats scientifiques. C'est le cas, notamment, du sophisme naturaliste, où un chercheur dérive ce qui devrait être de ce qui est. En d’autres termes, la science ne détient pas le monopole de la connaissance objective. Bien qu'il relève d'un autre type de discours, le travail philosophique entourant les valeurs n'est pas réduit à la subjectivité.

\section{I.2. LA NEUTRALITÉ AXIOLOGIQUE COMPRISE COMME UN IDÉAL ÉDUCATIONNEL}

Contre cette interprétation épistémologique, on peut penser que c'est la défense de l'autonomie des étudiants qui poussent Weber à formuler le critère de neutralité éthique ${ }^{5}$. Ainsi, la neutralité éthique ne serait pas la conséquence de considérations épistémologiques, mais plutôt d’un idéal éducationnel.

5 Une idée que Weber résume très bien dans le passage suivant: «Pourtant, il faut considérer non seulement leurs intentions [des professeurs], mais aussi la manière dont l'attitude qu'ils légitiment de leur autorité agit forcément sur une génération » (1965 [1917], p. 480). 
L'enseignement et l'entreprise scientifique en général révèlent des relations sociales. Pour Weber, l'université allemande correspond, par analogie, à une forme d'aristocratie. Une élite - le corps professoral - dispose d'une foule de pouvoirs de contrainte sur un groupe social, en l'occurrence les étudiants et les apprentis. Par exemple, on attend d'une salle de classe qu'elle soit silencieuse, à l'écoute du professeur (Weber, 1965 [1917], p. 478). En outre, le professeur dispose de plusieurs instruments pour s'assurer que l'étudiant apprenne et retienne la matière. L’on peut citer, en particulier, le contrôle des modalités d'évaluation, un statut d'expert érudit et une tribune privilégiée. Enfin, le poste de professeur implique une grande autorité morale sur les étudiants et les apprentis. Toutes les conditions sont réunies pour qu'une relation sociale aristocratique se forme entre l'étudiant et son professeur (McCarthy, 200I, p. I48-I49).

Cela ne signifie pas forcément que la transmission du savoir se fasse de haut en bas par contrainte. Il n'existe pas nécessairement une structure aristocratique au sein de la classe. Bien des professeurs créent toutes les conditions propices au débat fécond et ouvert en classe. Un professeur qui présenterait des «perspectives alternatives » et encouragerait l'évaluation critique pousserait ses étudiants dans une situation d'autonomie. Ce professeur délaisserait alors le «paternalisme» de la salle de cours décrit par Weber ${ }^{6}$. Ce que Weber souligne, c'est essentiellement que le professeur dispose d'une foule de moyens suffisants pour hiérarchiser la classe. Ainsi, un professeur peut décider, sans consultation, de couper court au débat et d'imposer ses vues. Sans que la classe soit nécessairement un lieu de soumission, il est clair que le professeur détient tous les instruments et règles institutionnelles pour créer de telles conditions (Whimster, 1980, p. 372).

Dans ce contexte, la neutralité éthique de Weber vise la formation d'étudiants autonomes. En évitant les jugements de valeurs, le professeur évite d'exacerber l'aristocratie académique et ouvre une voie vers l'auto-actualisation et l'auto-formation de l’académicien. Par une utilisation adéquate de la science, le chercheur protège en quelque sorte l'agencialité politique des individus 
(Myers, 2004, p. 276). La citation suivante montre l'importance, chez Weber, de la possibilité d'un discours éthique critique et sans contrainte:

Dans l'amphithéâtre, où l'on fait face à ses auditeurs, ceux-ci doivent se taire et c'est au Professeur de parler, et je considère comme irresponsable d'exploiter cette situation dans laquelle les étudiants sont contraints, pour leur formation, de suivre le cours d'un Professeur et où il n'y a personne qui s'oppose à lui par la critique, afin de marquer ses auditeurs de son opinion personnelle au lieu [...] de les faire profiter de ses connaissances et de son expérience scientifique (Weber, 2003 [1919], p. 95).

Quelques précisions quant aux informations contenues dans cet extrait s'imposent. D’abord, pour Weber, la philosophie politique est analogue au débat politique sur un point crucial, à savoir la possibilité d'un examen libre et critique des thèses proposées. Par examen libre et critique, on entend une réflexion approfondie sur un objet. De plus, cette réflexion doit être menée sans contrainte et selon une procédure logique et rationnelle. Par exemple, lorsqu'est défendue la thèse politique $\mathrm{X}$, l’audience doit avoir la liberté de ne pas écouter, de poser des questions, de défendre un point de vue distinct, de questionner la cohérence des idées présentées, soit de mener un examen critique de la thèse X.

L'examen libre et critique des thèses proposées est une étape essentielle à toute philosophie éthique et politique. En effet, la philosophie politique vise à émanciper l'individu des opinions reçues, de rapports d'influence tels que la conformité sociale? . Au contraire de la conformité, le philosophe a comme objectif fondamental d'éviter tout rapport de soumission intellectuelle pour user de sa liberté et de son entendement. En ce sens, la possibilité d'un discours libre et critique constitue une condition de possibilité de la philosophie politique. Ainsi, une thèse normative qu'il est impossible de critiquer, adoptée sous la contrainte, ne peut jamais constituer une véritable philosophie politique ${ }^{8}$. Une telle thèse

7 Ici, conformisme fait référence à une relation sociale d'influence et de contrôle visant à ce qu'un ensemble d'individus se soumettent à une norme commune.

8 Weber adhère à l'idéal éducationnel allemand, par lequel le jeune intellectuel développera des méthodes et des connaissances en vue de se réaliser de façon autonome (Myers, 2004, p. 285 n. I). Ultimement, le jeune intellectuel doit être en mesure d'évaluer des thèses normatives par lui-même, sans obéir à une force extérieure. Pour Myers, les 
relèvera plutôt du dogme ou de l'opinion (Myers, 2004, p. 277). C'est pourquoi Weber affirme: «En effet, le doute le plus radical est le père de la connaissance. [...] Si l'on voulait faire de la chaire universitaire un lieu pour discussions pratiques sur des valeurs, il est manifeste qu’on serait obligé d'y tolérer sans entraves la libre discussion des questions de principe fondamentales» (1965 [1917], p. 482).

L'aristocratie académique du professeur crée toutes les conditions propices pour convaincre l'auditoire, l'influencer dans le but qu'il accepte une thèse normative. Dans ce type de hiérarchie, le but du professeur n'est pas d'encourager les individus à réfléchir par eux-mêmes de façon autonome, mais simplement à les convertir. C'est tout le contraire de la réflexion rationnelle. Comment pouvons-nous former des penseurs autonomes si nous cherchons simplement à les convaincre? Et donc, comment pouvons-nous favoriser l'autonomie individuelle dans un lieu aristocratique comme l’amphithéâtre universitaire?

Dans cette perspective, la neutralité éthique constitue surtout une forme de «déontologie du savant». Jean-Rodrigue Paré (1999, p. 106) affirme qu’un professeur qui respecte la neutralité éthique ne fait que se «refuser tout privilège » face à ses étudiants. Weber condamne les professeurs qui nient l'autonomie de leurs étudiants, que ce soit par des démonstrations passionnées, ou pire, par une confusion entretenue entre des jugements personnels et objectifs - «an unavowed mixture of objective and personal judgments [...] that might give the impression that the thinker is offering objective truth », selon les termes de Marianne Weber (1988 [1975], p. 317, cité par Swatos et Kivisto, I99I, p. I19). Dans ces conditions, la classe devient simplement un autre lieu politique où les individus en contrôle entretiennent des rapports d'influence (Myers, 2004, p. 277 ; Ringer, 1997, p. 132 ; Yamawaki, 2007, p. 2 10).

Cette interprétation explique pourquoi Weber privilégie l'étude des moyens au discours proprement normatif. L'étude des moyens en vue d'une fin ne nous force pas à accepter la fin, à penser qu'elle est porteuse de valeur normative. C'est à l'étudiant, par son propre entendement, d'accepter l'hypothèse normathéorie de la science (1965 [1917) et du Savant et le Politique (1963 [1919]). 
tive comme étant vraie ou fausse. L'étudiant pourra, dans un lieu public et ouvert au discours, procéder à un examen critique de l'hypothèse normative (Swatos et Kivisto, 199I, p. I20). «Moreover, this new potential agency for the academic type and his scientific production evolves without belittling the integrity of intellectual endeavors, a blunder, in Weber's assessment, that the Kathedersozialisten [Historical school of economics] had committed in privileging engagement 》 (Myers, 2004, p. 279).

Cette interprétation a toutefois des limites. Ce ne sont pas tous les professeurs qui utilisent leur classe pour influencer leurs étudiants sur les plans éthique et politique. En outre, qu'en est-il des équipes de recherche, des conférences et des publications scientifiques ? Ces lieux sont-ils visés par la neutralité éthique? Nous savons aussi que l'embauche de professeurs marxistes et anarchistes constitue, pour Weber, un enjeu majeur. Devrions-nous considérer les valeurs d'un professeur lors de son embauche? Si oui, sous quelles conditions? La neutralité éthique, telle que formulée ici, ne semble pas répondre directement à cette constellation de questions.

Le critère de neutralité éthique chez Weber n'est pas organisé selon un système, et c'est pourquoi l'appliquer à d'autres problèmes que le cours magistral n'est pas toujours possible. Pour parer à cette difficulté, nous proposerons un critère de neutralité éthique intimement lié au problème de l'usage privé de la raison chez Kant.

\section{I.3. NEUTRALITÉ ET FONCTION: UNE INTERPRÉTATION KANTIENNE DU CRITĖRE WÉBÉRIEN}

Chez Kant, l'usage public de la raison est l'ensemble des actes de l'entendement par un individu en tant que personne. Cette activité est destinée à un public au sens propre du terme, à savoir l'ensemble d'une collectivité sociale. Par exemple, lorsqu'un penseur écrit une lettre ouverte dans un journal ou publie un essai en son nom, son acte reflète l'usage public de sa raison. L'acte de l'entendement est public tant et aussi longtemps que l'individu agit en son nom : si l'individu s'exprime au nom d'une organisation (un membre du clergé interprétant la Bible, un fonctionnaire interprétant la loi, un soldat obéissant aux ordres, etc.), il ne s'agit plus d'usage public de la raison, puisque l'individu 
exprime les idées d'une autre entité que lui-même (Kant, I784, p.484-485). Le terme public peut donc porter à confusion, en ce sens qu'il signifie deux choses distinctes: d'une part, que le destinataire est public, et d'autre part, que le destinateur est un individu agissant en tant que personne, et non en tant que représentant d'un tiers ou d'une institution (Braeckman, 2008, p. 295).

Kant, by contrast, defines the participants of his public sphere as speakers who address what Herder calls an ideales Publikum, or what we might call a virtual audience [...]. The only other qualification required of aspiring participants in the public sphere is designated by a curious double negative : they must not speak from the officially restricted position of a "private" person (Von Mücke, 20Io, p. 65).

Si une organisation ou une entité morale régit l'usage de la raison d'un individu, il s’agit alors d’usage privé de la raison. «Privé» fait ici référence à l’idée de privation: au fait qu'une partie soit refusée, à un usage limité en fonction de certaines conditions ou contraintes. Lorsqu'une personne accepte de parler ou d’agir au nom d'un tiers, elle se prive de certaines libertés. Par exemple, lorsqu'un prêtre est mandaté pour exposer la doctrine chrétienne dans une église, il se voit confier certaines tâches précises desquelles il ne peut déroger.

La distinction entre usage privé et public de la raison nous permet d'étendre notre compréhension de la neutralité axiologique à un vaste éventail de situations, notamment à l'embauche et aux différents lieux de recherche. Comme Heinich (2006), nous pensons que c'est la fonction du professeur qui est ici en jeu, et non l'individu stricto sensu. L'objectif est d'interpréter le mandat du professeur en fonction d'une communauté morale régissant l'université. Cette communauté morale aurait un objectif double, soit I) d'assurer la transmission de connaissances scientifiques par l'intermédiaire de spécialistes, et 2) d'y arriver sans nier l'autonomie des chercheurs, étudiants et professeurs au sein de l'Université. Pour réaliser ces objectifs, le ou la spécialiste des sciences sociales disposera donc de ressources et d'instruments, mais ces instruments ne devront en aucun cas être utilisés contre les deux objectifs fondamentaux de l'Université. Le professeur serait donc limité à un usage privé de la raison. 
La mission du professeur est de transmettre le savoir et les méthodes proprement scientifiques à un ensemble d'étudiants et d'apprentis. Il agit donc à titre privé, en ce sens qu'il est mandaté par une communauté morale pour accomplir une action bien spécifique. Le professeur doit, de plus, respecter le principe d’autonomie caractérisant l'université. Pour accomplir ces deux missions fondamentales, le professeur se voit confier des instruments et des ressources : un auditoire captif, des évaluations et des mécanismes de contrôle comme les présences, un droit de parole dans un lieu donné, etc.

Comme nous l'avons vu, le respect de l'autonomie se traduit chez Weber par l'exigence de neutralité éthique. Or, cela ne signifie pas qu'à tout moment, il soit interdit au professeur de participer au débat normatif entourant les valeurs et les normes sociales. Il est bien clair que l'usage privé de la raison est restreint à des moments précis dans le temps. En outre, l'usage privé de la raison au moment $\mathrm{X}$ est exigé d'une personne si, au moment $\mathrm{X}$, elle utilise les ressources et les instruments de cette fonction privée. Ainsi, cela signifie que le professeur doit ou bien I) abandonner les instruments et les ressources du professeur pour participer à un débat proprement public, ou bien 2) utiliser les instruments et les ressources du professeur tout en respectant le principe de neutralité éthique.

Revenons maintenant aux problèmes qui nous intéressent, à savoir l'expression de la normativité dans des situations comme l'embauche, les lieux de débat et les lieux de recherche. L'idée qu'une personne ne devrait pas être embauchée en fonction de ses idéaux normatifs est omniprésente chez Weber (1908; 1917; 1919). On peut comprendre cette idée en fonction d'une interprétation kantienne de la neutralité axiologique. L’idée que l’on puisse rejeter une candidature en fonction des croyances ou des idéaux politiques du postulant est une violation de la liberté de conscience et de l'autonomie du futur professeur. En effet, il s'avère illégitime pour le professeur d'utiliser ses instruments et ses ressources contre l'autonomie intellectuelle d'un individu. Comme l'embauche est une ressource propre à la fonction de professeur, ce dernier se doit donc de respecter l'autonomie des personnes touchées par cet instrument. Ainsi, aucune embauche (ou obstacle à l'embauche) n’est légitime sur la base de croyances ou d'idéaux politiques (Frankel, 1977). 
La même logique s'applique aux différents lieux universitaires, à savoir les colloques, les conférences, les publications scientifiques, soit tous les lieux où le professeur s'exprime en tant qu'expert. La question que doit se poser l'individu est toujours la même : l'individu est-il dans une situation où ses ressources de professeur créent une relation de contrôle à l'endroit d'individus ou d'un groupe? Si c'est le cas, le professeur a l'obligation morale de limiter l'usage de sa raison. Par exemple, si un colloque est organisé et qu'il est libre à chacun de participer ou non, le professeur n’a pas à se gêner de défendre une thèse normative en conférence, puisque chacun est libre d'entendre, de critiquer les arguments présentés, ou de quitter la conférence.

Cette compréhension systématique de la neutralité éthique nous permettra d’aborder plusieurs critiques du critère de neutralité. En outre, plusieurs philosophes affirment que l'introduction de concepts éthiques denses en sciences sociales nous mène à rejeter la neutralité éthique. Et certains penseurs naturalistes rejettent même la distinction entre les faits et les valeurs, ce qui rendrait l'application de la neutralité axiologique impossible. Nous analyserons et proposerons des réponses à ces argumentaires dans la prochaine section.

\section{HILARY PUTNAM, AMARTYA SEN, ET LES SCIENCES SOCIALES NORMATIVES}

La présente section expose l'argument de Putnam, et ses effets sur le critère de neutralité éthique. Il s’agit d'un argument fréquemment utilisé par plusieurs auteurs pour remettre en cause la neutralité axiologique (Coenen-Huther, 2007 ; Gerring et Yesnowitz, 2006; Lecomte, 2012 ; Thacher, 2006). La forme générale de l'argument rappelle aussi la démarche de Strauss, puisqu'il consiste à rejeter la dichotomie entre les faits et les valeurs pour réhabiliter le rôle du discours normatif. Dans ces conditions, il y a lieu d'étudier la logique de Putnam et Sen en profondeur pour répondre, en définitive, à une foule d’arguments «réfutant» la neutralité axiologique. 


\section{I. PUTNAM ET LA RÉFUTATION DE LA DICHOTOMIE FAIT/VALEUR}

Putnam (2004 [2002], p. 28) critique le positivisme logique de Carnap et à la dichotomie fait/valeur défendue par ce courant. Dans la tradition du positivisme logique de Carnap, les faits ont une existence objective, alors que les valeurs ont un statut subjectif. Plus précisément, les énoncés éthiques, tout comme par ailleurs les énoncés métaphysiques, sont considérés comme des énoncés dénués de sens, n’ayant aucune signification. Pour les positivistes logiques, seuls les énoncés empiriquement vérifiables sont doués de sens. La science empirique constitue dès lors la discipline objective par excellence.

Pour réfuter l'argument proposé par Carnap, Putnam vise à démontrer que la science, entreprise objective par excellence, repose elle aussi sur des valeurs. Ainsi, si les valeurs sont subjectives et que la science repose sur des valeurs, comment pouvons-nous affirmer que la science est une discipline objective? Putnam vise alors à montrer que les valeurs ont leurs propres critères d'objectivité.

En quoi la science repose-t-elle sur des valeurs? Comment des concepts comme le bien ou le juste pourraient-ils sous-tendre l'entreprise scientifique? L'erreur serait ici de réduire le domaine des valeurs à l'éthique et à la philosophie politique. Putnam (200I, p. 295-296) fait en effet référence à des valeurs épistémiques plutôt qu'éthiques : la simplicité, la cohérence, la pertinence, la plausibilité, la parcimonie et même la beauté font notamment partie des valeurs fondamentales en science. «[L]es jugements de "cohérence”, de "plausibilité", de "simplicité", ceux qui portent sur le caractère "raisonnable", [...] sont des jugements de la forme de "ce qui doit être" lorsqu'on a affaire à un raisonnement» (2004 [2002], p. 39). Bien sûr, les valeurs épistémiques ne sont pas identiques aux valeurs éthiques, mais dans les deux cas, il sagit de concepts exprimant le «devoir être ». Rappelons que dans la conception positiviste du savoir les énoncés relevant du «devoir être » sont par nature invérifiables. Ainsi, si les valeurs sont un «non-sens» comme le pensent les positivistes logiques, alors la sélection des théories scientifiques repose sur un critère sans aucune signification logique ou empirique (Putnam, 200I, p. 30I). 
Comment une valeur peut-elle s'avérer objective sans l'existence d'une correspondance avec un objet physique ? Putnam propose une notion d'objectivité souple, basée sur un travail d'examen critique des valeurs. L'examen informé et critique des normes fait référence à une «réflexion intelligente» touchant les valeurs étudiées, en l'occurrence une réflexion basée sur la discussion entre différents points de vue, une démarche ouverte face à des thèses opposées, la formulation d'hypothèses et de présupposés clairs et identifiables, ainsi que la clarification des implications et des limites des thèses étudiées (Putnam, 2004 [2002], p. II3-I I5). Évidemment, cette liste n'est pas exhaustive, Putnam rejetant l'idée d'un critère unique garantissant l'assertabilité des jugements éthiques. Il n'en demeure pas moins qu'une réflexion est intelligente du moment où sont exclues des attitudes comme le parti pris, la fermeture d'esprit ou d'autres obstacles à la progression du dialogue. De plus, comme chez Weber, l'objectivité en éthique chez Putnam n’a de sens que dans le cadre d'une discussion libre et ouverte, faisant échec à des «méthodes telles que [...] l'autorité»(Putnam, 2004 [2002], p. II5).

Jusqu'ici, Putnam confirme en quelque sorte l'analyse de Weber. En outre, les deux auteurs défendent l'existence d'un rapport aux valeurs préalable à l'entreprise scientifique, ainsi que d'une sphère des valeurs ayant ses propres critères d’objectivité, à commencer par l'examen critique des thèses étudiées. Il n'en demeure pas moins que, pour Putnam, toutes les conditions sont désormais réunies pour repenser la place du discours normatif dans les sciences sociales. Plus précisément, le fait qu'il soit possible de construire des jugements éthiques objectifs ouvre la voie à la construction de concepts éthiques denses objectifs.

Rappelons d'abord ce que sont les concepts éthiques denses. Ce sont des propositions - au sens logique du terme - renfermant des éléments descriptifs et normatifs indissociables. Par exemple, le concept de cruauté renferme des éléments descriptifs (X cause une douleur à Y, X cause cette douleur volontairement, cette douleur n’est pas nécessaire, etc.) et des éléments normatifs (X cause du mal à Y, etc.). Ces éléments sont indissociables : on ne peut séparer les éléments descriptifs des éléments normatifs sans changer la signification du concept de cruauté. Par exemple, supposons que X cause une douleur à Y, que cette douleur est volontaire, et que cette douleur n’est pas nécessaire. Supposons 
en outre que nous n'avons aucune indication quant à la valeur normative de l'action de X. Dans ces conditions, nous ne pouvons pas en conclure que X est cruel. X pourrait, par exemple, causer une douleur en pratiquant une chirurgie pour sauver la vie de $\mathrm{Y}$. En outre, X pourrait pratiquer cette chirurgie sans anesthésie pour des raisons contingentes - l'urgence de la situation, une pénurie à l'hôpital, ou tout autre facteur ponctuel. Causer une douleur non nécessaire volontairement n'est donc pas identique au fait d'être cruel. Ainsi, les éléments descriptifs sont indissociables des éléments normatifs dans un concept éthique dense comme la cruauté (Putnam, 20I I [1990], p. 334-335).

Suivant la logique de Putnam, nous pouvons dès lors construire des concepts éthiques denses objectifs. Il suffit d'entrelacer des éléments descriptifs objectifs (des lois, des règles et observations empiriques ayant des qualités épistémiques manifestes et une correspondance à l'objet satisfaisante) à des éléments normatifs objectifs (des règles, valeurs et normes issues d'un examen critique, d'une réflexion intelligente). Logiquement, le concept qui résulterait d'un tel entrelacement serait lui aussi objectif. Dans ces conditions, on pourrait facilement penser qu'il relève de la mission scientifique que d'élaborer un savoir à la fois descriptif et normatif, puisqu'il n’y aurait pas de frontière épistémologique (entre objectivité et subjectivité) pour ces différents niveaux de discours.

\subsection{SEN, LES CAPABILITÉS ET LA VALEUR DE LA SCIENCE}

À partir la conception objective de l'éthique développée par Putnam, l'économiste Amartya Sen a justifié la pertinence scientifique du concept de «capabilité », un concept éthique dense visant à introduire des éléments normatifs en sciences économiques (Putnam, 2004 [2002], p. 57 ; Sen, 2009, p. 309-3II). Les capabilités chez Sen font référence à «la liberté réelle que l’on a de choisir entre différentes façons de vivre» (2009, p. 279). Plus une personne dispose de liberté réelle, plus elle démontre des capabilités. Il existe des individus ou des groupes disposant d'une plus grande liberté réelle de choisir entre différentes options. Par exemple, les femmes ont longtemps été exclues du marché du travail et des fonctions politiques. À certaines époques, les hommes avaient donc une plus grande capabilité que les femmes. De plus, comme on le constate dans 
l'exemple précédent, la question des capabilités ne se résume pas à la question des ressources matérielles. Deux personnes peuvent disposer des mêmes moyens monétaires, mais ne pas avoir les mêmes capabilités. Il est même possible qu'une personne très pauvre dispose d'une liberté réelle d’agir, alors qu'une personne très riche subit de nombreuses contraintes (Sen, 2009, p. 28I-283, p. 309).

L'étude des capabilités englobe donc l’analyse des contraintes matérielles, sociales, psychologiques et autres à la liberté réelle, mais examine aussi les mécanismes pour éliminer ces obstacles (Sen, 2009, p. 284-286, p. 315-317). Un tel type d'analyse fait appel à une compréhension de plusieurs concepts à la fois normatifs et descriptifs comme le confort, la liberté et la contrainte qui constituent, notamment, des concepts éthiques denses (Sen, 2009, p. 283-285).

L’intérêt de Sen pour les capabilités est scientifique. Sur le plan de l'économie du bien-être, il s'agit d'un enrichissement notable par rapport aux approches «neutres» basées uniquement sur le PNB ou le PIB. Dans l'approche standard, la richesse des nations est déterminante pour mesurer le bien-être. Or, Sen vise à montrer qu'un État pauvre du point de vue du PIB n'implique pas que le bien-être y est moindre. Sans nier qu'il existe un lien entre bien-être et PIB, Sen pense que le lien entre capabilités et bien-être est davantage pertinent, en plus d'offrir un meilleur potentiel de prédictibilité (Dussault, 2008, p.7I). Sur le plan du comportement économique, Sen affirme que les agents économiques orientent leur comportement en fonction des capabilités. Ces dernières ne seraient donc pas seulement pertinentes du point de vue normatif, mais constitueraient aussi un facteur explicatif majeur. En d'autres termes, on pourrait mieux comprendre et prédire les choix des agents en étudiant leurs capabilités (Sen, 1999, p. 2I-24). L'étude des capabilités illustre en quelque sorte les avantages scientifiques possibles à mener une analyse à la fois descriptive et normative. Certains concepts éthiques denses présentent des qualités épistémiques comme la fécondité, la pertinence et la prédictibilité. En intégrant des concepts éthiques épais, le discours économique aurait une plus grande valeur scientifique (Sen, 2009, p. 285-286). 
Nous avons donc deux arguments distincts auxquels la neutralité éthique doit répondre: d’une part, comment concilier les qualités épistémiques des concepts éthiques denses au respect de la neutralité éthique? D’autre part, comment défendre un traitement distinct pour l'éthique au sein des lieux académiques si cette discipline, au même titre que les autres sciences, est objective et rigoureuse? La prochaine section tente de déterminer s'il est possible et souhaitable de concilier les qualités épistémiques des concepts éthiques denses à l’analyse wébérienne de la neutralité axiologique.

\section{PEUT-ON CONCILIER LA NEUTRALITÉ AXIOLOGIQUE AUX OBJECTIFS DE SEN ET PUTNAM?}

L’argument soulevé par Putnam et Sen nous mène d’abord à reconnaître que, dans certains paramètres institutionnels, la discussion à la fois descriptive et normative est possible et souhaitable. La frontière entre science et philosophie morale n'est en effet plus infranchissable. Il est possible d'imaginer des concepts objectifs entrelaçant un contenu descriptif et normatif.

Il serait donc tout à fait possible d'inaugurer une nouvelle discipline objective, à la fois normative et descriptive, qui aurait comme caractéristique de tendre vers une certaine forme d'objectivité assouplie. Des organismes de recherche œuvrant dans la sphère publique pourraient remplir cette fonction : mener une discussion normative sur la base de données scientifiques et de théories morales. Ces organismes pourraient contribuer significativement à la discussion publique sur les politiques sociales. Cela implique qu'une discipline adoptant une méthode scientifique pourrait disposer de ses propres institutions, sans pour autant enfreindre la neutralité axiologique.

Pour clarifier notre argument, il y a lieu de rappeler les conditions de l'objectivité des normes en éthique. Pour Putnam, c'est l'examen critique d’une norme qui lui confère un certain degré d’objectivité. En outre, plus le travail critique entourant une norme est vaste et exhaustif, plus notre compréhension de cette norme tend vers l'objectivité. Le critère d'objectivité est similaire chez Weber. Pour Weber, l'étude des normes doit se faire de façon libre et critique, 
sans quoi on ne peut véritablement prétendre à l'objectivité philosophique. Dès lors qu'une personne dispose d'instruments de persuasion pour imposer un certain discours normatif, il s'agit alors de relations sociales telles que l'influence et l'endoctrinement, et non d'une recherche proprement philosophique.

Alors, pourquoi est-ce que les institutions scientifiques œuvrant dans la sphère publique respecteraient le critère de neutralité axiologique? De façon générale, l'idée suggérée ici est que, pour respecter la neutralité axiologique, des groupes œuvrant dans la sphère publique n'ont pas à respecter les mêmes contraintes que les individus entretenant des relations sociales d'influence ou de contrôle. La neutralité axiologique est ici comprise comme un mécanisme visant à défendre l'autonomie des différents membres de la communauté universitaire. Nous avons suggéré que la possibilité d'un examen libre et critique est une condition fondamentale à cette autonomie. Dans la sphère publique, les institutions ou les individus ne peuvent pas bloquer la tenue de cet examen par des mécanismes ou des ressources (à moins que cet espace public ne le soit pas vraiment). Dès lors, si les groupes de recherche soumettent leurs énoncés normatifs à un examen critique ouvert, il n’y a pas violation de la neutralité axiologique. Ces lieux de recherche respecteraient donc le critère de neutralité axiologique parce qu'ils œuvrent dans la sphère publique.

Si la neutralité éthique de Weber marque les limites de la discussion normative dans les lieux sous l'autorité du professeur, il est toujours possible d'imaginer des lieux où cette discussion est possible et souhaitable. La distinction entre les lieux de délibération publique et les lieux politiques reste toutefois très importante. Qu'un organisme mène une discussion à la fois descriptive et normative dans un lieu de délibération public, c'est une chose. Or, cela n'autorise pas n'importe quelle personne en position d’autorité à faire la promotion d'une théorie morale dans un lieu où le débat est limité par des instruments de contrôle. L'usage privé de la raison doit être contenu si l'on veut préserver l'autonomie intellectuelle des agents. 
Une chose est donc bien claire chez les deux auteurs : une thèse normative imposée ne peut jamais constituer un discours véritablement objectif. Dans ces conditions, une authentique recherche objective des normes bonnes ou mauvaises ne peut se faire sous l'autorité d'un professeur. C'est pourquoi, pour être cohérent avec la nécessité d'un examen critique en éthique, on doit accepter le critère de neutralité axiologique formulé par Weber.

\section{I. UNE ILLUSTRATION EMBLÉMATIQUE DE CE QUE DOIT SIGNIFIER UNE SCIENCE PUBLIQUE}

Pour clarifier davantage ce que signifie l'évolution d'institutions scientifiques dans la sphère publique, nous proposons une illustration emblématique. Le type d'institution que nous proposons pour les sciences sociales est déjà décrit par de nombreux auteurs dans différents champs disciplinaires. Patrick Gaudray (20I0), par exemple, s'insurge contre la construction et la modification des normes de santé par la communauté scientifique seule. L’auteur propose de partager et d'échanger en société sur la construction de ces indicateurs. Un autre exemple important de cette conception de la science est à trouver dans certaines épistémologies féministes, notamment la «standpoint theory». Pour des auteurs comme Susan Hekman (1997, p. 357), Helen Longino (1990) ou Sandra Harding (I99I, p. I42), il est nécessaire, pour éviter les accusations de subjectivité et de relativisme, de construire des standards de preuve partagés et publics. Le fait que ces critères soient publics les expose à la critique, une condition de l'objectivité forte défendue par ces auteures (Hekman, 1997, p. 354).

Nous avons choisi de présenter l'approche d'Alain Desrosières, touchant la construction des indicateurs publics en statistique (PIB, chômage, IPC, indices d'équité, performances étatiques, etc.). De par sa portée (la construction d'indicateurs quantitatifs est le fait de toutes les disciplines humaines), l’argument de Desrosières s'applique tant à l'économie, la sociologie, la science politique qu'aux approches féministes. En d'autres termes, le caractère général de cet argument nous semble approprié pour illustrer une conception publique des différentes sciences sociales. 
Dans son dernier essai Prouver et gouverner. Une analyse politique des statistiques publiques (20I4), Alain Desrosières étudie les conditions d'objectivité dans la construction des quantifications statistiques. Quantifier consiste à «faire exister sous une forme numérique ce qui, auparavant, était exprimé par des mots et non par des nombres » (Desrosières, 20I4, p. 38). Au moins une convention est à l'origine de la quantification. Prenons le cas du chômage. Une personne qui occupe un emploi moins de dix heures par semaine estelle sans emploi ? Et qu'en est-il si elle travaille I heure par semaine ? Est-ce qu'une personne invalide doit entrer dans le calcul du chômage? Et quid de l'invalidité temporaire? Parle-t-on d'invalidité temporaire sur une période d'une semaine, de trois mois, de cinq ans ? (ibid., p. 78).

Cela signifie que les acteurs doivent s'entendre, échanger, comparer leurs conceptions de l'objet pour lui attribuer des caractéristiques quantifiées. Le chômage est l'illustration emblématique d'une quantification menée par des économistes. Mesurer suppose plutôt que l'objet en question se présente déjà sous une forme mesurable. À supposer que l'on dispose déjà d'une unité de mesure commune (comme le mètre ou le yard), aucune convention n'est nécessaire pour mesurer la superficie d'un parc ou la hauteur d'une montagne.

Il existe des indicateurs statistiques à la fois descriptifs et normatifs. La variation du chômage, par exemple, nous indique un changement de fait, mais aussi une amélioration ou une dégradation de la situation de l'emploi. Bien que Desrosières n'emploie pas cette expression, de nombreux indicateurs statistiques sont des concepts éthiques denses. Ces indicateurs sont donc des conventions, construites par des groupes, qui reflètent la valeur normative d'une situation. Par exemple, il serait surprenant qu'une personne affirme:«le chômage est en forte hausse, ce qui signifie que la situation de l'emploi s'améliore ». On dira de cette personne qu'elle ne comprend pas ce qu'est le chômage, ou à tout le moins, qu’elle ne définit pas le chômage comme les autres membres de la collectivité.

Or, qui a le pouvoir de décider ce qui entre dans la définition du chômage? Le fait que cet indicateur échappe à la délibération publique le rend fort suspect aux yeux de plusieurs citoyens: on le soupçonne d'être biaisé, notamment parce qu'on ne peut participer à construire cet indicateur. Puisque les citoyens 
ne peuvent se prononcer sur ce qui, d'un point de vue normatif, devrait constituer le chômage, cet indicateur perd une grande part de sa légitimité.

Alors, comment s'assurer que des indicateurs comme le chômage, qui sont à la fois descriptifs et normatifs, conservent leur légitimité ? Desrosières suggère que c'est par une approche intersubjective qu'une statistique publique acquiert légitimité et neutralité :

Pour qu'une statistique joue son rôle social de référent supposé neutre, au-dessus des parties d'un conflit ou des groupes sociaux, elle doit être instituée, garantie par des procédures démocratiques elles-mêmes légitimes. Elle contribue alors à faire de la réalité et non pas simplement à la «refléter» [...]. [Cette idée] ne nie pas l'existence de l'inflation ou du chômage. Mais elle attire l'attention sur le fait qu'inflation et chômage peuvent être pensés, exprimés, définis et quantifiés de multiples façons (2014, p. 75).

Les indicateurs qui sont produits à la suite d'une délibération collective, d'un exercice dialogique, ont une plus grande objectivité. Leur construction est transparente, fait suite à un examen critique, et permet un échange éclairé entre différents intervenants. Au contraire, des indicateurs comme le chômage font l'objet de vives critiques, précisément parce que la construction de ces indicateurs n'est pas transparente (ibid., p. 78-79).

Comme la quantification d'un phénomène social comme le chômage échappe aux règles de la délibération collective, la confiance en cet indicateur est affaiblie. «La relative malléabilité de la définition du chômage alimente les soupçons, toujours présents, de manipulations gouvernementales» (ibid., p. 79). Desrosières défend ici une conception publique de la science. Si des indicateurs statistiques sont normatifs, il faut s'assurer que leur contenu évaluatif soit soumis à un examen libre et critique. 


\section{CONCLUSION}

L'hypothèse initiale sur laquelle repose cet article était qu'il existe des conditions de possibilité propres au discours éthique, et que la neutralité axiologique constitue, en définitive, un devoir pour quiconque incarne l'objectivité de respecter ces conditions de possibilité. Chez Weber, le professeur ou la professeure s'exprimant dans une classe forcée au silence, à l’abri de la critique, ne peut affirmer sérieusement rechercher l'objectivité en éthique. Comme la recherche de l'objectivité est ce qui distingue l'éthique d'autres types de discours - comme l'endoctrinement ou des relations publiques - il s'agit en quelque sorte d'une condition nécessaire. Si l'on recherche véritablement l'objectivité normative, on doit accorder à ses «interlocuteurs» le droit de parole, l'examen libre et critique des thèses présentées. Ou encore, on doit présenter ses idées dans un lieu public, ouvert à la discussion.

C'est sur ce constat, plutôt concret, que se base la neutralité axiologique chez Weber. Nous avons voulu généraliser cette idée, dans le but de l'appliquer à une vaste gamme de problématiques. En effet, le critère de neutralité éthique chez Weber n'est pas organisé selon un système, et c'est pourquoi l'appliquer à d'autres problèmes que le cours magistral n'est pas toujours possible. Pour ce faire, nous avons repris l'analyse kantienne de la distinction entre usage public et privé de la raison. Lorsqu'il est en fonction, le professeur doit respecter les objectifs de la communauté universitaire. La neutralité éthique est donc pensée selon la fonction de professeur et à l'usage privé de la raison. Grâce à cette logique, nous avons pu déterminer quel est l'usage légitime de la raison dans diverses circonstances, notamment dans les séminaires, les publications scientifiques, les colloques et l'embauche.

Une analyse de la neutralité axiologique aurait été incomplète sans approfondir les critiques contemporaines de la dichotomie fait/valeur, et du supposé impact de cet argumentaire sur la neutralité éthique. Pour Putnam et Sen, la réfutation d'une dichotomie entre les valeurs «subjectives» et les faits «objectifs » remet en cause la nécessité de séparer, dans les sciences, le discours descriptif du discours normatif. Putnam montre en effet comment les normes 
peuvent atteindre un certain degré d'objectivité. L'examen informé des normes constitue, à long terme, une façon de tendre vers l'objectivité en éthique. En d'autres termes, plus une norme fait l'objet d'une analyse critique, plus notre compréhension de cette norme et de sa valeur tend vers l'objectivité.

Face à la critique de Putnam et Sen, nous répondons qu'on ne peut accepter ou réfuter la neutralité axiologique quel que soit le lieu. Weber pense au contraire le critère de neutralité axiologique en fonction de règles institutionnelles précises. Il existe des lieux où les règles institutionnelles permettent l'entrelacement des niveaux d'analyse descriptif et normatif, et d'autres lieux où c'est impossible de le faire sans violer l'autonomie des agents. Ainsi, une intégration normative aux sciences sociales doit se faire dans des cadres institutionnels donnés, où l'examen libre et critique est possible et encouragée.

Une science ancrée dans la sphère publique répond à cette demande. Nous avons illustré les raisons appuyant une cette conception de la science, notamment la transparence, l'objectivité, ainsi que des conditions fortes d'échange et de délibération. Une science sociale ancrée dans la sphère publique permet d'enrichir les concepts de la science, de préserver son objectivité, d'augmenter sa pertinence et son utilité, tout en protégeant l'autonomie intellectuelle de la communauté scientifique.

\section{BIBLIOGRAPHIE}

BEHNEGAR Nasser, 1997, «Leo Strauss's Confrontation with Max Weber:

A Search for a Genuine Social Science», The Review of Politics, 59-I, p. 97-125.

BOWEN Don R., 1977, «Objectivity as a Normative Standard», The Journal of Politics, 39-I, p. 20I-210.

BRAECKMAN Antoon, 2008, «The Moral Inevitability of Enlightenment and the Precariousness of the Moment: Reading Kant's "What Is Enlightenment?" 》, The Review of Metaphysics, 62-2, p. 285-306.

BRUUN Hans Henrik, 2007, Science, Values and Politics in Max Weber's Methodology, Hampshire, Ashgate Publishing Ltd. 
COENEN-HUTHER Jacques, 2007, «Classifications, typologies et rapport aux valeurs», Revue européenne des sciences sociales, XLV-138, p. 127-I40.

COLLIOT-THÉLÈNE Catherine, 2003, «Préface», in Le Savant et le Politique, Paris, La Découverte, p.9-59.

DESROSIÈRES Alain, 2014, Prouver et Gouverner. Une analyse politique des statistiques publiques, Paris, La Découverte.

DUSSAULT Antoine, 2008, «Putnam et la critique de la dichotomie fait/valeur. La critique de quoi au juste?», Mémoire présenté à la Faculté des études supérieures de l'Université de Laval, Québec.

FRANKEL Charles, 1977, «Facts, Values, and Responsible Choice», in The Ethics of Teaching and Scientific Research, S. Hook, P. Kurtz et M. Torodovich (éds), Buffalo, Prometheus Books, p. 23-28.

GAUDRAY Patrick, 2014, «Éthique, lieu de conscience, d'interrogation et de débat», in Science et Société. Les normes en question, Michelle Bergadaa et al. (éds), Actes Sud.

GERRING John et YESNOWITZ Joshua, 2006, «A Normative Turn in Political Science?», Polity, 38-I, p. 101-133.

GONTHIER Frédéric, 2006, «Relativisme et vérités scientifiques chez Max Weber», L’Année sociologique, 56-I, p. 15-39.

HARDING Sandra, 1991, Whose Science? Whose Knowledge? Thinking from Women's Lives, Ithaca, Cornell University Press.

HEINICH Nathalie, 2006, «La sociologie à l'épreuve des valeurs», Cahiers internationaux de sociologie, 121-2, p.287-315.

HEKMAN Susan, 1997, «Truth and Method: Feminist Standpoint Theory Revisited», Signs, 22-2, p. 341-365.

HENNIS Wilhem, 1994, «The Meaning of 'Wertfreiheit' on the Background and Motives of Max Weber's "Postulate" », Sociological Theory, 12-2, p. I13-125.

LECOMTE Jacques, 2012, «Est-il justifié de parler de psychologie positive?», Les cahiers internationaux de psychologie sociale, I, p. 21-36.

LONGINO Helen, 1990, Science as Social Knowledge, Princeton, Princeton University Press. 
MCCARTHY George E., 200I, Objectivity and the Silence of Reason:

Weber, Habermas and the Methodological Disputes in German Sociology,

New Brunswick, Transaction Publishers.

MILLER Richard, 1979, «Reason and Commitment in the Social Sciences》, Philosophy \& Public Affairs, 8-3, p. 24I-266.

MITTLEMAN Alan, 1999, «Leo Strauss and Relativism: The Critique of Max Weber», Religion, 29-I, p. I5-27.

MYERS Perry, 2004, «Max Weber: Education as Academic and Political Calling», German Studies Review, 27-2, p. 269-288.

PARÉ Jean-Rodrigue, 1999, Les Visages de l'engagement dans l'oeuvre de Max Weber, Paris, L'Harmattan.

PORTIS Edward, 1980, «Political Action \& Social Science: Max Weber's Two Arguments for Objectivity», Polity, 12-3, p. 409-427.

PUTNAM Hilary, 1984, «The Craving for Objectivity», New Literary History, 15-2, p. 229-39

-, 2004 (2002), Fait-valeur: la fin d'un dogme et autres essais, trad. de M. Caveribère et J.-P. Cometti, Paris et Tel Aviv, Éd. de l'Éclat.

-, 201 I (1990), Le Réalisme à visage humain, trad. de Cl. Tiercelin, Paris, Gallimard.

RINGER Fritz, 1997, Max Weber's Methodology. The Unification of Cultural and Social Sciences, Cambridge (MA), Harvard University Press.

SEGADY Thomas William, 1987, Values, Neo-Kantianism, and the Development of Weberian Methodology, New York, Peter Lang Publishing.

SEN Amartya, 1999, Development as freedom, Oxford et New York, Oxford University Press.

-, 2009, L'Idée de Justice, trad. de P. Chemla, Paris, Flammarion.

STRAUSS Leo, 1999 (1965), Natural Right and History, Chicago, University of Chicago Press.

SWATOS William H. et KIVISTO Peter, 1991, «Beyond Wertfreiheit: Max Weber and Moral Order», Sociological Focus, 24-2, p. II7-128. 
THACHER David, 2006, «The Normative Case Study», American Journal of Sociology, III-6, p. I631-1676.

VON MÜCKE Dorothea, 2010, «Authority, Authorship, and Audience:

Enlightenment Models for a Critical Public», Representations, III-I, p. 60-87.

WEBER Max, 1965 (1917), «Essai sur le sens de la "neutralité axiologique" dans les sciences sociologiques et économiques», in Essais sur la théorie de la science, trad. de J. Freund, Paris, Plon, p. 475-526.

-, 2003 (1919), Le Savant et le Politique, trad. de C. Colliot-Thélène, Paris, La Découverte.

-, 2008 (1908), «The Bernhard Case », trad. de G. C. Wells, in Max Weber's Complete Writings on Academic and Political Vocations, New York, Algora Publishings, p. 53-58.

WEBER Marianne, 1988 (1975), Max Weber. A biography, trad. de H. Zohn, New Jersey, Transaction Publishers.

WHIMSTER Sam, 1980, «The Profession of History in the Work of Max Weber: Its Origins and Limitations», The British Journal of Sociology, 3I-3, p. 352-376.

YAMAWAKI Naoshi, 2007, «Rethinking Weber's Ideal-Types of Development, Politics, and Scientific Knowledge», in Max Weber's "Objectivity" Reconsidered, L. McFalls (éd.), Toronto, University of Toronto Press, p. 206-224. 